\title{
Quantum mechanics teaching resources from the Institute of Physics
}

\section{Antje Kohnle ${ }^{1}$ \& Derek Raine ${ }^{2}$}

${ }^{1}$ School of Physics and Astronomy, University of St Andrews, St Andrews KY16 9SS, UK

${ }^{2}$ Centre for Interdisciplinary Science, University of Leicester, University Road, Leicester LE1 7RH, UK

\section{Corresponding author:}

Antje Kohnle, School of Physics and Astronomy, University of St Andrews, St Andrews KY16 9SS, UK Email: ak81@st-andrews.ac.uk

\begin{abstract}
In December 2013, the Institute of Physics (IOP) launched a set of freely available resources at quantumphysics.iop.org for the teaching and learning of quantum mechanics. The website includes about 80 short articles written by experts in the field and 17 interactive simulations with accompanying activities for an introductory course in quantum mechanics starting from two-level systems. The articles are arranged according to five themes, including a focus on quantum information, interpretations of quantum mechanics, the mathematical structure of the theory, physics applications and historical experiments. The resources make topics such as entanglement, hidden variables and quantum information theory accessible to introductory-level students. They can be used flexibly for a variety of instructional aims at both the introductory and more advanced level. The website includes links to pre-readings, suggestions for further reading, a glossary of technical terms and allows users to rate their understanding of articles.
\end{abstract}

Sharing of these resources is encouraged, with all usage under the Creative Commons CC BY-NC-ND licence. Solutions to problems and activities are available for instructors by emailing quantumphysics@iop.org. Instructors interested in evaluating these resources with their students in order to help us further develop and optimize the site are requested to contact the corresponding author.

Keywords: quantum physics, 2-level systems, simulations, quantum information

\section{Introduction}

Quantum theory has a reputation for being difficult to grasp and removed from real-world problems. 
Many studies have documented student difficulties with quantum mechanics and their underlying reasons (see, for example, Domert et al. 2005, Wittmann et al., 2005, Zhu \& Singh 2012). The Institute of Physics (IOP) has developed a set of free educational resources available at quantumphysics.iop.org that challenges this stereotype. The resources offer a new introductory quantum curriculum centred on two-level systems as well as more traditional material to support undergraduate physics students and instructors (Kohnle et al. 2014a). The need for these resources emerged at an IOP discussion meeting in December 2010 led by Derek Raine on the teaching of quantum mechanics that brought together instructors across the UK (IOP 2012).

Developing quantum theory using two-level systems (such as a spin $1 / 2$ particle, a photon in an interferometer, an atom with two energy levels) can have multiple advantages: It allows from the start the discussion of experiments that have no classical analogue and focus on key concepts such as superposition and quantum uncertainty; it allows from the start a discussion of interpretations of quantum mechanics and quantum information theory; and it is mathematically less challenging than the traditional wave mechanics approach, requiring only elementary linear algebra rather than differential equations and calculus. The IOP resources include much of the linear algebra needed for this approach. While two-level systems and associated topics such as entanglement, hidden variables and quantum information are commonly discussed in advanced quantum mechanics textbooks, there are very few resources available for the introductory level and arguably none that are tailored to UK courses. The IOP resources aim to fill this gap and give instructors and students the resources needed to discuss these topics both at the introductory and the more advanced level.

The resources include about 80 short articles centred on questions, problems, a glossary of technical terms and 17 interactive simulations with accompanying activities. Figure 1 shows a screenshot from the website. Texts have been authored by researchers in quantum information theory (Pieter Kok, Sheffield and Dan Browne, UCL) and foundations of quantum mechanics (Mark Everitt, Loughborough). Interactive simulations and activities were developed by Antje Kohnle (St Andrews), building on the expertise of the QuVis project (www.st-andrews.ac.uk/physics/quvis). They were coded by a team of four undergraduate students at St Andrews. Articles went through a rigorous editorial process by Derek Raine (Leicester) and Elizabeth Swinbank (York). The project was led by Derek Raine and Christina Walker (project manager at (OP).

\section{Overview of the resources}

\section{Content}

The content is organized into five themes: informational, with a focus on quantum information; foundational, with a focus on interpretations of quantum mechanics; mathematical, with a focus on the mathematical structure of quantum mechanics; physical, with a focus on physics applications and a more traditional wave mechanics approach; and historical, with a focus on historical experiments leading to the development of quantum mechanics. Each theme presents an ordered path through the material. Core articles are included in all themes, others are specific to only a subset of themes or a single theme. This approach allows the resources to be used flexibly for a variety of instructional aims, both for the introductory and the more advanced level. At the University of St Andrews, we have used parts of the IOP resources with our first year, second year and fourth year students. This included using articles as pre-lecture reading assignments and using simulations in computer classroom workshops, as in-class demonstrations and homework assignments. Simulations enable students to learn new concepts as well as consolidate understanding of concepts.

As one example, in what follows we describe the informational theme. This theme starts with an illustration how classical concepts break down using the behaviour of single photons in a Mach-Zehnder interferometer. Single photon interference is first described qualitatively and then mathematically. The theory is expanded using spin $1 / 2$ particles and atoms with two energy levels as other examples of two-level systems. The theme includes applications such as atomic clocks and gravitational wave detection. This is followed by two-particle states, entanglement and quantum information, including articles on the no-cloning theorem, quantum cryptography, quantum teleportation and quantum computing. The theme ends with a transition to continuous systems.

\section{Simulations}

Interactive simulations can help students to engage with and explore physics topics through high levels of interactivity, prompt feedback, and multiple representations of physics concepts (Podolefsky et al., 2010, Clark \& Mayer 2011). Through the careful design of interactive controls and their ranges, students can be implicitly guided in their exploration. The IOP resources include 17 interactive simulations suitable both for the introductory and more advanced levels, covering the topics of linear algebra, single photon interference, fundamental quantum mechanics concepts, the Bloch sphere representation, entanglement, hidden variables and quantum information. Figure 1 shows one of these simulations. 


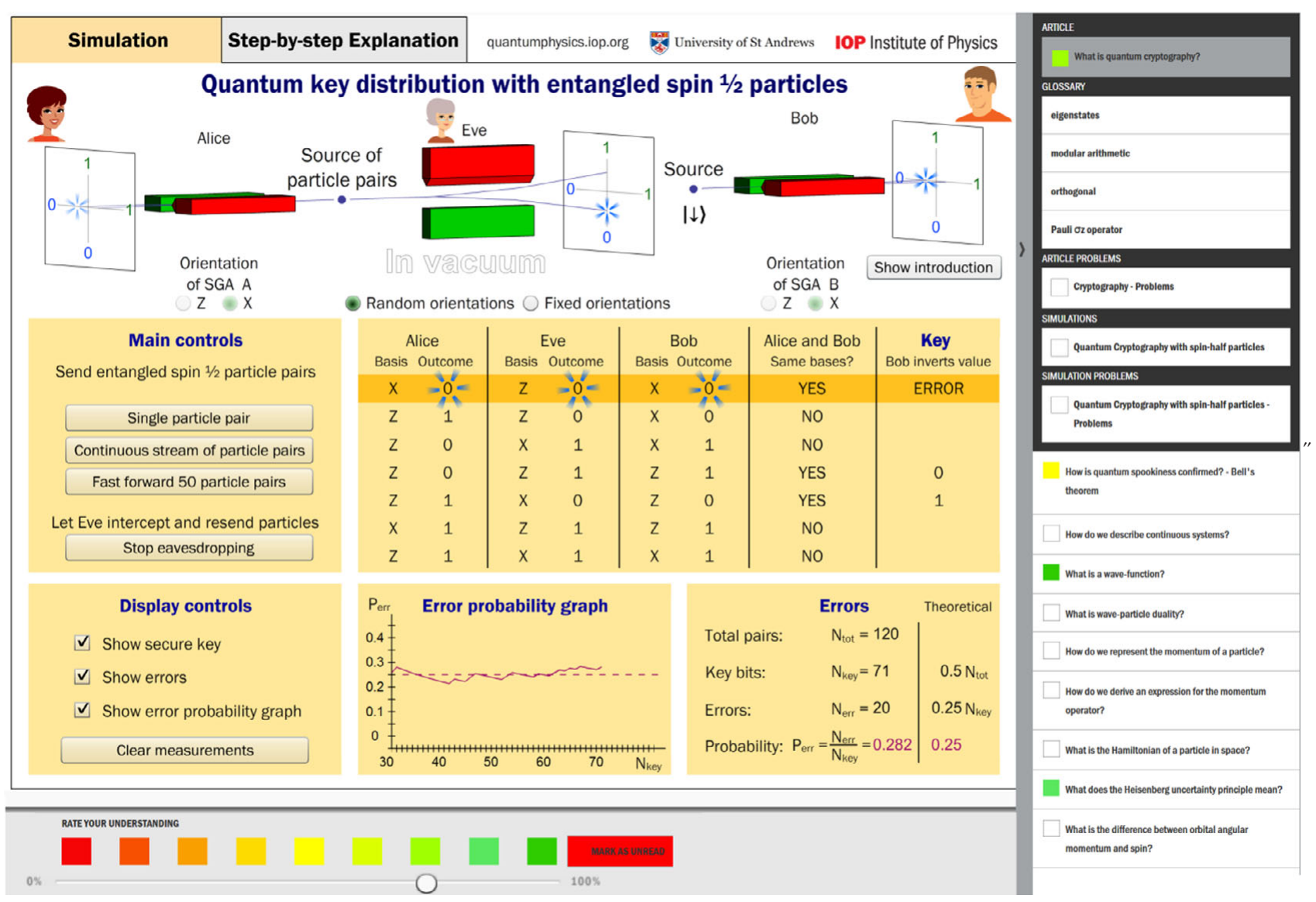

Figure $1 \mathrm{~A}$ screenshot of the "What is quantum cryptography?" article, showing a simulation embedded in the article, the slider (below the simulation) for users to rate their understanding of the article, and the navigation panel (right-hand side) that lists articles, shows users' ratings and includes glossary terms and links to problems.

The simulations are research-based, and evaluation with students plays a key role in their development. Simulations and activities were iteratively refined using individual student observation sessions for each simulation, where students freely explore a simulation and then work on the associated activity (Kohnle et al. 2014b). For a subset of simulations, we also carried out in-class trials. Each simulation comes with an activity aimed to promote guided exploration and sense-making and help students progress from simpler to more complex situations. Instructors can request solutions to the activities as well as the article problems by emailing quantumphysics@iop.org. Simulations are also available for download from the QuVis website www.st-andrews.ac.uk/physics/quvis.

\section{Website structure}

The website is freely available, but requires users to register. Registration allows the site to save difficulty ratings entered by the user, and to display these ratings as well as the articles already read via coloured squares in the navigation panel (see figure 1). This panel also includes a glossary of technical terms and, if available, links to article problems and the simulation activity (see figure 1). The navigation panel can be minimized, particularly useful for viewing articles on small screens. The top of most articles includes links to recommended pre-requisite articles, and shows the user via the colour-coding scheme whether they are have already read and understood these pre-requisite articles. The texts include links to glossary terms, other articles and simulations where applicable. A number of articles include links to further reading. The website settings allow users to choose their preferred text size and provide help on navigation.

\section{Future plans}

Further revision of the website is ongoing. Next steps include the development and optimization of the content and functionality of the website informed by student and instructor feedback. If you are interested in helping us evaluate these resources at your institution, please contact the corresponding author. We would provide support for implementation as well as short pre- and post-tests to assess student learning gains and survey questions to elicit student feedback.

\section{Acknowledgements}

We thank the Institute of Physics for funding this project and developing and maintaining the quantumphysics.iop.org website. 


\section{References}

Clark, R.C. and Mayer, R.E. (2011) E-Learning and the science of instruction. San Francisco: John Wiley.

Domert, D., Linder, C. and Ingerman, A. (2005) Probability as a conceptual hurdle to understanding one-dimensional quantum scattering and tunnelling. European Journal of Physics 26 (1), 47-59. doi:10.1088/0143-0807/26/1/006.

IOP (2012) IOP Higher Education Group Newsletter $11,6-7$.

Kohnle, A., Bozhinova, I., Browne, D., Everitt, M., Fomins, A., Kok, P., Kulaitis, G., Prokopas, M., Raine, D. and Swinbank, E. (2014a) A new introductory quantum mechanics curriculum. European Journal of Physics 35 (1), 015001-1-9. doi:10.1088/0143-0807/35/1/015001.

Kohnle, A., Baily, C., Hooley, C. and Torrance, B. (2014b) Optimization of Simulations and Activities for a New Introductory Quantum Mechanics
Curriculum. In Proceedings of the 2013 Physics Education Research Conference, Portland (eds. P.V. Engelhardt, A.D. Churukian and D.L. Jones), pp209-212.

Podolefsky, N.S., Perkins, K.K. and Adams, W.K. (2010) Factors promoting engaged exploration with computer simulations. Physical Review Special Topics Physics Education Research 6 (2), 020117-1-11. doi:10.1103/PhysRevSTPER.6.020117.

Wittmann, M.C., Morgan, J.T. and Bao, L. (2005) Addressing student models of energy loss in quantum tunnelling. European Journal of Physics 26 (6), 939-950. doi:10.1088/0143-0807/26/6/001.

Zhu, G. and Singh, C. (2012) Improving students' understanding of quantum measurement. I. Investigation of difficulties. Physical Review Special Topics Physics Education Research 8 (1), 010117-1-8. doi:10.1103/PhysRevSTPER.8.010117. 